Proceedings

\title{
Case presentation \& tygacil (tigecycline) usage
}

\section{Case I-Mr. Rassam}

a) 24 year's old, male, and medically free, from Yemen.

b) Admitted on $8 / 4 / 2014$

i. With a 1-month Hx. of Gunshots to Chest/Abdomen that was operated on in Yemen.

ii. After 1-month post laparotomy Lt Nephrectomy Splenectomy Rt Nephrostomy Tracheostomy patient.

iii. Was treated with unknown medications \& unknown antibiotics.

\section{O/E}

I. $\mathrm{CAO}^{*} 3$

II. Temp 37.5 - Pulse 125/m - RR 16/m - BP 123/70 - O2\% 97\%

III. Ill Looking, cachectic, Pale, Jaundiced.

IV. Tracheostomy in situ.

V. Bilateral Harsh Breathing SoundsDecreased AEB.

VI. Soft Abdomen with previous midline incision of previous surgery \& 3 drains:-

i. Lt UQ à Bile

ii. Rt Loin à Urine

iii. Lt Loin à Empty Figure 1 \& Table 1

Additional Admission Labs

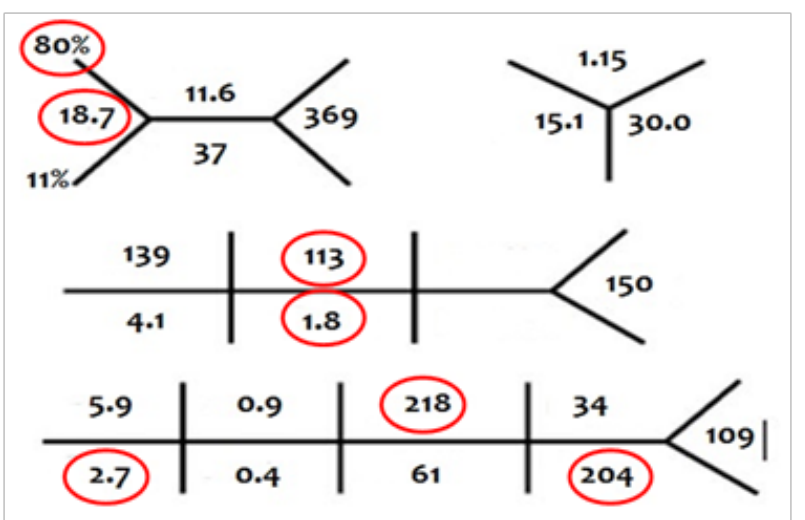

Figure I Soft Abdomen with previous midline incision of previous surgery.

\section{Chest+abdomen+pelvic CT scan (oral contrast)}

i. Bilateral pleural effusion \& more on RT side associated with atelectasis \& ground glass opacities bilaterally with peri-bronchial thickening \& LT fissural effusion.

ii. Translucent tubular shadow from LT lung extending to the SC tissue (fistula). Drainage tubes in upper abdomen $\left(1^{\text {st }}\right.$ one @ porta
Volume 2 Issue 4 - 2015

\author{
Kamel Al Mashaqi \\ General Surgery Resident, Jordan
}

Correspondence: Kamel Al Mashaqi, General Surgery Resident (PGY 4), TIH, Jordan,

Email Kamel.Muhammad@gmail.com

Received: June 25, 2015 | Published: August 14,2015

iii. Ascites

iv. Double J Catheter in RT Kidney -UB.

\section{Management of case}

i. NPOIV Fluids (Resuscitation)

ii. TPN Protocol

iii. I/O Charting

iv. Bld Urine Sputum Nasal Cx

v. Labs \& Radiology (CXR+ Pan CT Scan Nephrostogram)

vi. Pre-Op assessment \& evaluation (PRBCs FFP)

vii. Contact Isolation

viii. IV Medications (Tygacil Meropenem Diflucan Nexium Clexane Hydrocortisone Perfalgan)

Table I Admission labs

\begin{tabular}{llll}
\hline Lab & Value & Lab & Value \\
\hline CRP & $58.9 \uparrow$ & Urine Analysis & Prt+1 \\
ESR & $44 \uparrow$ & & Glu+3 \\
PO4 & 4.3 & Bld +3 \\
$\mathrm{Mg}$ & $1.42 \downarrow$ & & Pus-Numerous \\
$\mathrm{Ca}$ & 9 & & $\begin{array}{l}\text { Red-Nume- } \\
\text { rous }\end{array}$ \\
$\mathrm{Nasal} \mathrm{Cx}$ & Acinetobacter & Bld Cx & - -ve \\
Sputum Cx & Acinetobacter & Urine Cx & -ve \\
\hline
\end{tabular}

\section{Consultations to}

i. Cardiologist

ii. Nephrologist 

iii. Pulmonologist
iv. Urologist Surgeon
v. Infectious Disease Specialist

\section{| 0/4/20 | 4 ( 2 days post admission)}

Ist Surgery (Redo exploratory laparotomy with LT thoracotomy approach)
i. LT Lung decortications LT Lung Bullet injury repair.
ii. Gastro-Pleuro-Cutaneous Fistula excision.
iii. Primary repair (double layer) of stomach.
iv. LT hemicolectomy End Ileostomy formation.
v. Feeding Jejunostomy Tube Insertion.
vi. Pancreatic Necrosectomy.
vii. LT Chest Tube2 free abdominal drains insertion.

\section{0/4/20 I 4 ( 2 days post admission)}

\section{I $^{\text {st }}$ Surgery (Urology Surgery)}

i. RT Ureteroscopy

ii. DJ Insertion

\section{Post Op Day (0) to Day (3)}

i. Patient transferred back to ICU-Surgical with same pre-op management.

ii. Patient started on Enteral feeding by (Jejunostomy tube) with ensure milk.

iii. Patient remained (Tachycardiac+Feverish).

\section{Post Op day (4)- I 4/4/20 I 4}

i. We discovered wound dehiscence with fluid discharge from abdominal wound.

ii. $2^{\text {nd }}$ Surgery $\left(2^{\text {nd }}\right.$ laparotomy+Wound Repair with Component separation closure technique).

iii. Tissue Cultures obtained \& (+ve for Staphylococcus sppCoagulase Negative) \& ONLY sensitive to tigecycline.

\section{Post Op day (4)- | 4/4/20 | 4}

i. Patient transferred back to ICU-Surgical Intubated on Ventilator (atelectasis of LT lung \& poor expansion with bad ABG's).

ii. Patient kept on same protocol (IV Fluids TPN Protocol Enteral feeding I/O Charting IV Medications (Tygacil Meropenem Diflucan Nexium Clexane Perfalgan).

iii. We added (Octreotide) to our list of medications for 5 days.

iv. Patient remained (Tachycardiac Feverish).

\section{Post Op day ( I 0)-20/4/20 I 4}

i. We discovered (by clinical \& radiological evidence) a leak at the site of feeding Jejunostomy tube. ii. $3^{\text {rd }}$ Surgery (laparotomy+Repair).

iii. Same Pre-Op management but stopped the enteral feeding for few days.

iv. Patient remained (Tachycardiac + Feverish).

\section{Post op day ( I 8)-28/4/20 I 4}

i. Patient fully extubated with Spontaneous Breathing after several trials over the past few days.

ii. Multiple interval blood \& other Cx came back -ve.

iii. Oral fluid feeding resumed for the $1^{\text {st }}$ time from initial trauma with success.

iv. Vital signs were near NORMAL for 48hours.

v. Patient was able to ambulate for $1^{\text {st }}$ time from initial trauma.

Post op day (19)-29/4/20 I 4

i. Ventilator stopped \& Tracheostomy removed.

ii. Tygacil with Meropenem stopped \& patient Started on Piperacillin/ Tazobactam.

iii. Kept on Vancomycin.

iv. Stopped feeding by Jejunostomy tube.

v. Kept in ICU-Surgical with oral fluid feeding \& observation of multiple spikes of fever \& Tachycardia.

\section{Post op day (23)-03/05/20 I 4}

i. Jejunostomy feeding tube removed.

ii. CT Chest/Abdomen/Pelvis (Normal Study).

iii. Kept in ICU - Surgical with oral fluid feeding \& observation of multiple spikes of fever \& Tachycardia.

\section{Post op day (24)-04/05/20 I 4}

i. Non ionic contrast meal (Normal Study).

\section{Post op day (27)-07/05/20 I 4}

i. Piperacillin/Tazobactam changed to Tienam.

ii. TPN stopped \& Full regular diet given.

iii. CT Pulmonary Angio done (Normal Study).

iv. Kept in ICU-Surgical with oral fluid feeding \& observation of multiple spikes of fever \& Tachycardia.

\section{Post op day (32)- I 2/05/20 I 4}

i. Patient transferred to floor.

ii. Regular diet (High Protein) \& Oral medications.

iii. Fully ambulating.

\section{Post op day (44) - 24/05/20 I 4}

i. Patient discharged home.

ii. Patient came back to near normal level of activity \& independence. 
iii. V/S was normal for $>48 \mathrm{hrs}$.

iv. WBC \& CRP went down to near normal levels.

v. All Cx came back -ve.

vi. All Radiological Studies came back as normal studies.

\section{Case 2-Mr. Qannaff}

i. 20 year's old, male, and medically free, from Yemen.

ii. Admitted on 04/02/2014-NO formal Hx - Per Reports

iii. With a 1-week Hx. of High Velocity Gunshots to Abdomen that was operated on in Yemen.

iv. After 1-week post laparotomy+2 drains found inside abdominal cavity with multiple visceral injuries (liver/pancreas/duodenum/ gastric/IVC vs. Portal??).

v. Was treated with unknown medications \& unknown antibiotics.

\section{O/E}

i. CA but disoriented.

ii. Paraplegic

iii. Temp 37.2-Pulse 120/m-RR 31/m-BP 132/90- $\mathrm{O}_{2} \%$ 98\%

iv. Ill looking, cachectic, Pale but NOT Jaundiced.

v. Bilateral Harsh Breathing Sounds+Decreased AE @ Basal Rt.

vi. Tender Abdomen+previous midline incision of previous surgery+bullet inlet @, RT Para midlineoutlet @, LT lumbar \& 2 drains:-

\section{Rt loin à Bile}

Rt Loin à Bile Figure 2 \& Table 2

Additional Admission Labs

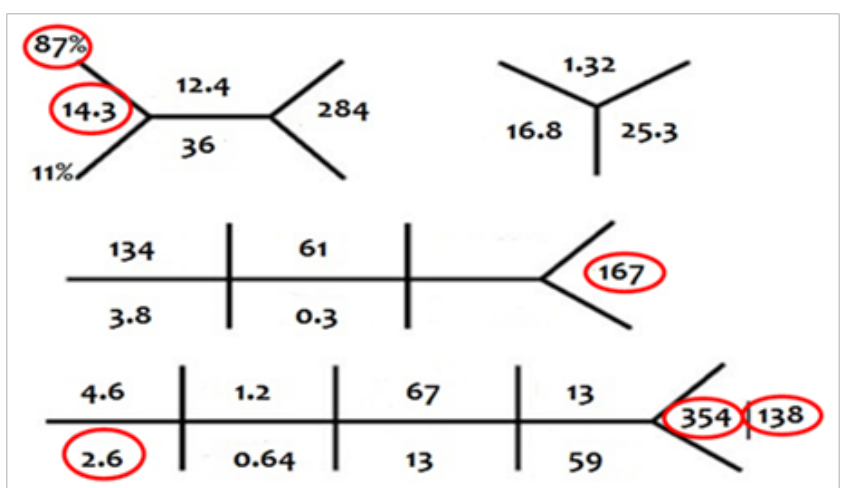

Figure 2 Tender Abdomen+previous midline incision of previous surgery+bullet inlet @ RT Para midlineoutlet @ LT lumbar.

\section{Chest+abdomen+pelvic CT scan (triple contrast)}

\section{Lumbosacral MRI}

i. L2 vertebral fracture with injury to cord.
Table 2 Admission labs

\begin{tabular}{llll}
\hline Lab & Value & Lab & Value \\
\hline $\mathrm{Mg}$ & $1.9 \downarrow$ & Urine Analysis & Prt+1 \\
$\mathrm{Ca}$ & $7.4 \downarrow$ & & Glu+1 \\
& & & Bld +4 \\
& & & Pus-4 -6 \\
& & & Red-Numerous \\
Nasal Cx & ESBL & Bld Cx & -ve \\
Sputum Cx & ESBL & Urine Cx & -ve
\end{tabular}

\section{Management of case}

\section{Intubation+Full sedation}

i. NPOIV Fluids (Resuscitation)

ii. TPN Protocol

iii. I/O Charting

iv. Bld Urine Sputum Nasal Cx

v. Labs \& Radiology (CXR+Pan CT Scan)

vi. Pre-Op assessment \& evaluation (PRBCs FFP)

vii. Contact Isolation

viii. IV Medications (Meropenem Nexium Clexane)

\section{Consultations to}

i. Neurosurgeon

ii. Pulmonologist

iii. Infectious Disease Specialist

iv. ENT Surgeon

\section{5/02/20 I 4 (I days post admission)}

i. $1^{\text {st }}$ Surgery (Exploratory laparotomy) with

ii. Resection of distal stomach duodenum head of pancreas.

iii. Retroperitoneal exploration \& evacuation of multiple bilomas.

iv. CBD Tube drainage.

v. 2 free abdominal drains inserted with 2 VAC dressings.

vi. Multiple packs inserted.

vii. Findings (Type V complex pancreatic-duodenal injuries).

\section{Post Op day (0) to day (2)}

i. Patient transferred back to ICU-Surgical with same pre-op management.

ii. Blood transfused with FFP in regular basis. 
iii. Vancomycin added to $\mathrm{Rx}$ regimen.

iv. Sandostatin added to $\mathrm{Rx}$ regimen.

v. Clexane changed to Hibor.

vi. Patient remained (Tachycardiac Feverish).

\section{Post Op day (3)-08/02/20 I 4}

i. $\quad 2^{\text {nd }}$ Surgery (Laparotomy+RemovalofPacking + Gastrojejunostomy + choledochojejunostomy + pancreaticojejunostomy).

ii. Patient transferred back to ICU-Surgical Intubated on Ventilator.

iii. Patient kept on same protocol (IV Fluids $+\mathrm{I} / \mathrm{O}$ Charting+IV Medications (Meropenem+Vancomycin+Nexium+Hibor).

iv. Patient remained (Tachycardiac+Feverish).

\section{Post Op day (7)- I 2/02/20 I 4}

i. $3^{\text {rd }}$ Surgery (WoundExploration+Debridement+Dressing+Removal of VAC Dressing).

ii. Sputum Cx (+ve for Acinetobacter)

\section{Post Op day (I I)- I 6/02/20 I4}

i. $4^{\text {th }}$ Surgery (Wound Exploration+partial closure with vicryl mesh+component separation technique).

ii. $\mathrm{D} / \mathrm{C}$ drains (2)

\section{Post Op day (13)- | 8/02/2014}

i. $5^{\text {th }}$ Surgery (Exploratory Laparotomy+Retroperitoneal drainge of subpancreatic fluids + Dressing+ + progressive closure).

ii. Tissue $\mathrm{Cx}$ (+ve for Acinetobacter)

iii. Colistin added to the $\mathrm{Rx}$ regimen.

iv. Extubated on $\mathrm{O}_{2}$ mask.

v. Patient remained (Tachycardiac+Feverish).

\section{Post Op day ( I 5)-20/02/20 I4}

i. Patient started on (Ensure Milk by NGT+Apple Juice).

\section{Post Op day ( I 7)-22/02/20 I4}

i. $6^{\text {th }}$ Surgery (Closure of abdominal wall).

ii. Blood $\mathrm{Cx}$ (+ve for Acinetobacter) \& Tygacil added to the $\mathrm{Rx}$ regimen.

iii. Re-intubated due to Respiratory Distress.

\section{Post Op day ( I 8)-23/02/20 | 4}

i. Enteral feeding started.

ii. D/C Chest Tube.

iii. Meropenem stopped.

iv. Flagyl added to the Rx regimen.

v. Patient remained (Tachycardiac+Feverish).
Post op day (20)-25/02/20 I 4

i. $7^{\text {th }}$ Surgery (Wound lavage under GA+Dressing).

ii. CXR à white LT lung due to collapse.

iii. Patient is still intubated.

iv. Blood $\mathrm{Cx}(-\mathrm{ve})$.

v. Sputum $\mathrm{Cx}$ (+ve Acinetobacter).

vi. TPN Started.

vii. Patient remained (Tachycardiac+Feverish).

\section{Post Op day (22)-27/02/20 I4}

i. Trial of Extubation à Failed.

ii. $8^{\text {th }}$ Surgery (Tracheostomy+DUGA).

Post Op day (24)-0 I/03/20 I4

i. $\mathrm{D} / \mathrm{C}$ Ventilator.

ii. $\mathrm{V} / \mathrm{S}$ was normal for $>48 \mathrm{hrs}$.

iii. All Cx came back -ve.

\section{Post op day (26) - 03/03/20 I4}

i. Patient discharged to Yemen, AMA by MEDEVAC.

ii. Patient is considered a HIGH risk for non-professional management with risk of death but AMA.

\section{Why tygacil?}

i. Tygacil (tigecycline) has in vitro activity against a wider range of pathogens

ii. Resistant Gram +v: Enterococcus faecalis (VRE), Enterococcus faecium (VRE), Staphylococcus aureus (MRSA), Staphylococcus epidermidis (MRSE)

iii. Resistant Gram -ve: Acinetobacter baumannii, E. Coli, Klebsiella pneumoniae, Stenotrophomonas maltophilia, Tygacil is not affected by (ESBLs).

iv. Atypicals: New- Legionella pneumophila.

Tygacil (tigecycline) has in vitro activity against a wider range of pathogens

i. Anaerobes: Bacteroides (distasonis, fragilis, ovatus, thetaiotaomicron, uniformis, vulgatus), Clostridium perfringes, others.

ii. Gram +ve: Enterococcus (avium, casseliflavus, faecalis, faecium, gallinarum), Staphylococcus (aureus, epidermidis, haemolyticus), Streptococcus (pyogenes, agalactiae, anginosus grp).

iii. Gram -ve: Aeromonas hydrophila, Citrobacter (freundii, koserr), Enterobacter (cloacae, aerogenes), E.Coli, Klebsiella (oxytoca, pneumoniae) Serratia marcescens, Pasteurella multocida.

Tygacil (tigecycline) has in vitro activity against a wider range of pathogens

i. Gram +ve: New-Streptococcus Pneumoniae, including cases with concurrent bacteremia 
ii. Gram -ve: New-Haemophilus influenzae \& Parainfluenzae (Figure $3 \&$ Table 3).

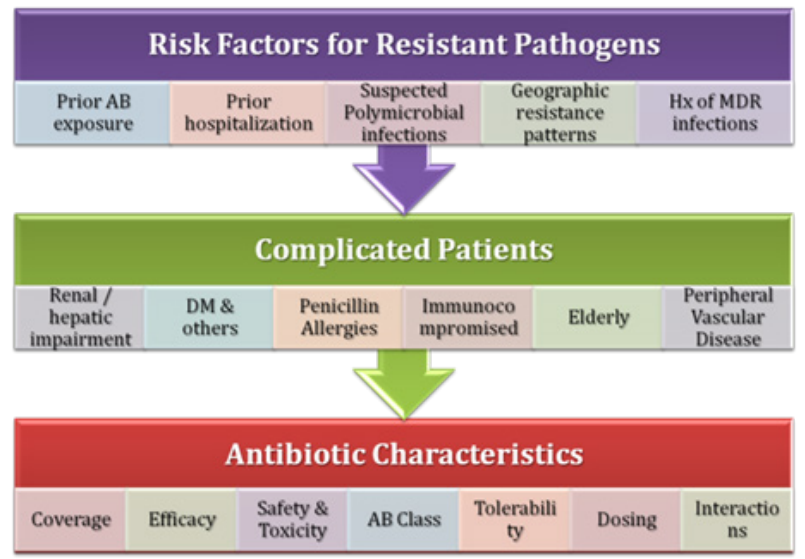

Figure 3 IV Antibiotic choice.

\section{Why Tygacil?}

i. Clinical coverage: Expanded broad-spectrum coverage including resistant gram positive, resistant gram negative, and anaerobes

ii. Efficacy: Proven as empiric mono therapy in patients with cIAI.

iii. Dosing Regimen: Does not require dosage adjustments for patients Table 3 Admission labs with renal impairment regardless of severity. No adjustments with mild-to-moderate hepatic impairment.

iv. Drug Interactions: Low potential for drug-drug interactions

v. Results: Efficacy in treatment

vi. Convenient: Q $12 \mathrm{hr}$ dosing

\section{Infectious diseases society of america guidelines for treatment of clAl}

Optimal dosing: To ensure maximum efficacy \& minimal toxicity \& to reduce antimicrobial resistance, for empiric Rx of cIAI, guidelines suggest $100 \mathrm{mg}$ initial dose of tigecycline, followed by $50 \mathrm{mg}$ every $12 \mathrm{hrs}$.

cIAI: community acquired infections: Guidelines recommend tigecycline as single-agent $\mathrm{Rx}$ for initial empiric $\mathrm{Rx}$ in adults with infections of mild-to-moderate severity \& perforated or abscessed appendicitis.

Treatment duration: a) According to guidelines, antimicrobial therapy should be limited to 4-7days, unless it is difficult to achieve adequate source control.

i. The recommended duration of Rx with Tygacil for cIAI is 5 to 14days.

\begin{tabular}{|c|c|c|c|c|c|}
\hline & Tygacil & $\begin{array}{l}3^{\text {rd }} 4^{\text {th }} \\
\text { Cephalosporin }\end{array}$ & Carbapenems & Fluroquinolones & Pipa/Tazo \\
\hline Gram +ve & $\underline{V}$ & $\sqrt{ }$ & $\sqrt{ }$ & $\sqrt{ }$ & $\sqrt{ }$ \\
\hline Gram -ve & $\underline{\sqrt{ }}$ & $\sqrt{ }$ & $\sqrt{ }$ & $\sqrt{ }$ & $\sqrt{ }$ \\
\hline Atypicals & $\underline{\sqrt{ }}$ & 0 & 0 & $\sqrt{ }$ & 0 \\
\hline Anaerobes & $\underline{\sqrt{ }}$ & 0 & $\sqrt{ }$ & 0 & $\sqrt{ }$ \\
\hline R. Gram +ve & $\underline{V}$ & 0 & 0 & 0 & 0 \\
\hline R. Gram -ve & $\underline{v}$ & 0 & $\sqrt{ }$ & 0 & 0 \\
\hline Pseudomonas & 0 & 0 & $\sqrt{ }$ & 0 & $\sqrt{ }$ \\
\hline
\end{tabular}

\section{Acknowledgements}

\section{Conflict of interest}

None.

The author declares no conflict of interest. 